\title{
Resources available for autism research in the big data era: a systematic review
}

\author{
Reem Al-jawahiri ${ }^{\text {Corresp., }}{ }^{1}$, Elizabeth Milne ${ }^{1}$ \\ 1 Psychology, University of Sheffield, Sheffield, United Kingdom \\ Corresponding Author: Reem Al-jawahiri \\ Email address: rabdalsahib1@sheffield.ac.uk
}

Recently, there has been a move encouraged by many stakeholders towards generating big, open data in many areas of research. One area where big, open data is particularly valuable is in research relating to complex heterogeneous disorders such as Autism Spectrum Disorder (ASD). The inconsistencies of findings and the great heterogeneity of ASD necessitate the use of big and open data to tackle important challenges such as understanding and defining the heterogeneity and potential subtypes of ASD. To this end, a number of initiatives have been established that aim to develop big and / or open data resources for autism research. In order to provide a useful data reference for autism researchers, a systematic search for ASD data resources was conducted using the Scopus database, the Google search engine, and the pages on 'recommended repositories' by key journals, and the findings were translated into a comprehensive list focused on ASD data. The aim of this review is to systematically search for all available ASD data resources providing the following data types: phenotypic, neuroimaging, human brain connectivity matrices, human brain statistical maps, biospecimens, and ASD participant recruitment. A total of 33 resources were found containing different types of data from varying numbers of participants. Description of the data available from each data resource, and links to each resource is provided. Moreover, key implications are addressed and underrepresented areas of data are identified. 
1

2

3

4

5

6

9

10

11

Resources Available for Autism Research in the Big Data Era: A Systematic Review

\author{
Reem Al-jawahiri ${ }^{1 *}$, Elizabeth Milne ${ }^{1}$
}

5

${ }^{1}$ Department of Psychology, University of Sheffield, Sheffield, South Yorkshire, United (1) Kingdom.

8

9

${ }^{*}$ Corresponding author

Email: $\underline{\text { Rabdalsahib1@sheffield.ac.uk }(R A) ~}$ 


\section{Abstract}

13 Recently, there has been a move encouraged by many stakeholders towards generating big,

14 open data in many areas of research. One area where big, open data is particularly valuable is in

15 research relating to complex heterogeneous disorders such as Autism Spectrum Disorder (ASD).

16 The inconsistencies of findings and the great heterogeneity of ASD necessitate the use of big and

17 open data to tackle important challenges such as understanding and defining the heterogeneity

18 and potential subtypes of ASD. To this end, a number of initiatives have been established that

19 aim to develop big and / or open data resources for autism research. In order to provide a useful

20 data reference for autism researchers, a systematic search for ASD data resources was conducted

21 using the Scopus database, the Google search engine, and the pages on 'recommended

22 repositories' by key journals, and the findings were translated into a comprehensive list focused

23 on ASD data. The aim of this review is to systematically search for all available ASD data

24 resources providing the following data types: phenotypic, neuroimaging, human brain

25 connectivity matrices, human brain statistical maps, biospecimens, and ASD participant

26 recruitment. A total of 33 resources were found containing different types of data from varying

27 numbers of participants. Description of the data available from each data resource, and links to

28 each resource is provided. Moreover, key implications are addressed and underrepresented areas

29 of data are identified.

\section{$31 \quad 2$ Introduction}

32 Recently, there has been a move towards generating 'big' and 'open' data in many areas of

33 science such as psychology, neuroscience, genetics, and omics. The move is driven by many 
34 stakeholders involved in the academic research process, including funding bodies, publishers,

35 researchers, and participants. Funding bodies (including the Medical Research Council, the

36 Wellcome Trust, and the US National Institutes of Health (NIH), among others) are increasingly

37 funding large studies and initiatives that facilitate data sharing. In addition, their data-policies are

38 recommending and, in certain cases, requiring the studies that they fund to submit a data-sharing

39 plan and to share their data. Publishers are also playing a role in driving big data and data sharing

40 efforts through their data policies, data-oriented special issues and call for papers [e.g. 1-2], and

41 the launch of machine-readable data journals (e.g. Scientific Data and GigaScience). Depending

42 on the data type and the journal, researchers are expected to make their data available either at

43 the time of publication or after an embargo period. Researchers are also playing a role by getting

44 involved in data-sharing initiatives. Some are increasingly preparing their data for sharing (e.g.

45 through proper data management and annotation) and/or requesting access to data from others

46 for secondary analysis. Although participants have concerns about data-sharing policies, many

47 are also supportive of this drive and are participating in studies and initiatives that ask them to

48 consent for their data to be used and shared somewhat flexibly to different researchers and

49 studies [3-4].

51 'Big data' is an evolving term and its use to refer to data in the mentioned areas is relatively

52 new. Therefore, in this review, the term 'big data' is used to broadly refer to datasets that are

53 larger than what researchers conventionally handle in their respective fields. While 'open data'

54 refers to data that is available for researchers to access without a fee or with a reasonable fee

55 relative to the many costs of collecting data (e.g. funds, time, resources, etc.) 
One area where big data is particularly valuable is in research relating to complex

58

heterogeneous disorders such as Autism Spectrum Disorder (ASD). ASD is a heterogeneous neurodevelopmental disorder (or spectrum of disorders, [see 5]) that is diagnosed based on a dyad of core behavioural symptoms of social communication deficits and characteristic repetitive and restricted behaviour [6]. Although these two core symptom domains somewhat unify and define ASD, there is significant variability between people with ASD in terms of symptom severity, symptom profile, level of cognitive function, and comorbidities [7]. This heterogeneity is potentially a major factor impacting on the rate of replication of ASD studies, and is leading some researchers to "give up on a single explanation for autism" [8] and others to propose the possibility that ASD should not be considered as a single disorder [5]. Instead, they suggest that within ASD, there could be groups of distinct disorders with many aetiologies [5]. Given that the cause, the aetiology and the genetic landscape of ASD remain unknown, developing a clear picture of exactly what ASD is, and what it is not, is increasingly important.

\section{Indeed, ASD is phenotypically heterogeneous between and within affected individuals who} show varying trajectories and response to treatment [9-11]. Heterogeneity is also implied in ASD's genetic architecture: non-overlapping genetic risk factors are continuing to reveal locus and allelic heterogeneity. Many different genes are involved with ASD and the genetic findings seem to vary depending on numerous factors such as the sample's phenotypic profile, gender [12], pedigree (e.g. simplex or multiplex families) [13-14], and sample size, etc. With the advent of next generation sequencing techniques, the number of genes found that are associated with ASD is increasing to over 800 genes [15]; consequently, it is becoming even more challenging to find unified explanations and functional associations between the genes involved. Thus, the 
80 unique nature of ASD's heterogeneity and the current state of autism research highlights the

81 importance of investigating the possibility that ASD may be a disorder with different subtypes,

82 which have distinct phenotypic, cognitive, and/or genetic profiles.

83

84 A number of studies have attempted to define the subtype structure of ASD by searching for 85 empirically derived clusters of participants within relatively large datasets. The majority of work 86 in this area has taken a phenotype-first approach, i.e. by attempting to identify potential ASD 87 subtypes by clustering participants based on behavioural, cognitive or medical characteristics 88 [16-18]. In some cases, attempts have been made to demonstrate the convergent validity of these potential subtypes using genetic data [19-20]. However, this work has yielded varying findings with regard to the number of identified subgroups, the classification of phenotypes, and the resulting identified genes or loci. For example, the number of proposed potential subgroups 92 varies from two [20-21], three [16,22], four [18-24], to six [25]. In addition, there is no agreed description of subtype markers for ASD as there are many relevant possible categories and specifiers [26]; potential candidates include: symptom severity [16,17,20,22,24], insistence on sameness scores [27-28], IQ scores [21,29], sensory features [30-31], and ASD comorbidities [18]. Although stratification attempts have been shown to improve statistical power in individual

97 genetic studies, e.g. leading to higher LOD scores in linkage studies [29,32-33], replicable 98 findings are somewhat rare such that different studies find different sets of ASD-implicated 99 genes and loci [e.g. 29,32-33]. In addition, Chaste et al. [34] found that phenotypic stratification

100 had only a small effect on increasing power and the homogeneity of genetic findings. However 101 in this case phenotypic stratification was based on rather broad, and univariate characteristics 
102 such as symptom severity, IQ and diagnostic category, which, in isolation, may not be

103 representative subtype markers.

104

105 Contrary to the phenotype-first approach, the genotype-first approach is driven by a reverse

106 strategy in which participants are grouped and studied based on a shared genetic etiology (e.g., a

107 specific CNV or gene associated with a disorder) as opposed to a common phenotypic or clinical

108 profile (e.g., language impairment, ASD diagnosis) [35-36]. Following the identification of a

109 genetically homogenous group, assessments are made to deeply characterize the respective group

110 (i.e., phenotypic characterization and otherwise) and understand its association with the relevant

111 disorders if applicable. Among the recent studies related to the ASD subtyping literature are

112 those supported and facilitated by the Simons Foundation Autism Research Initiative (SFARI),

113 which adopted the genotype-first approach to studying individuals with 16p11.2 CNVs (a

114 recurrent CNV significantly associated with ASD) [see 37-38]. Deep characterization of $16 \mathrm{p} 11.2$

115 CNV carriers whether through examining their phenotypic and clinical features [e.g., 39-40],

116 electrophysiological brain activity as recorded with M/EG [e.g., 41-42], or otherwise [e.g., 43-

$11744]$ would ultimately establish whether a particular genetically homogenous group could be

118 regarded as an empirically-derived ASD subtype. As of yet, the ASD subtyping literature,

119 phenotype- and genotype-centric, is growing and advancing in its approach and methodology to

120 investigating ASD potential subtypes; this is largely due to consortia and data resources sharing

121 ASD and ASD-related data to the research community.

122

123 Although this is not meant to be a comprehensive review of the ASD heterogeneity /

124 subtyping literature, from the aforementioned studies and findings it could suggest that 
125 successful identification of potential ASD subtypes, if they are to be found, requires data from

126 large numbers of participants who are drawn from a wide sample of the autism spectrum. The

127 task of assembling such a cohort may often be unfeasible for a single researcher, or team of

128 researchers, to accomplish. Therefore, motivated, in part, by the role that big, and open, data can

129 play in defining the typology of ASD, and in understanding the neural aetiology and genetic

130 origin of ASD, a number of initiatives have been established, primarily in the USA, that aim to

131 develop big data resources for autism research. One example is SFARI, which is an ASD data

132 resource that shares data from large numbers of specific phenotypic and genetic profiles of

133 participants, and allows different researchers with different expertise to conduct various analyses

134 on the same sample [e.g., 45]. In addition to the large datasets provided by SFARI, numerous

135 other open ASD datasets exist for use by the research community.

136

137 The aim of this article is to identify and describe available resources that provide access to

138 data obtained from participants with ASD. Several existing reviews have accumulated a general

139 list of available data resources, although these are not ASD-specific [46-50]. Although useful,

140 these lists are non-comprehensive and are mainly limited to neuroimaging resources. In addition,

141 some of the lists included resources that are inaccessible to researchers (e.g. limited access data

142 to only respective consortia members), and thus would not be beneficial to the research

143 community at large. Most importantly, to date, no detailed list of available sources of ASD data

144 exists. Therefore, in order to provide a useful data reference for autism researchers, a systematic

145 search was conducted, and the findings were translated into a comprehensive list focused on

146 ASD data. A total of 33 resources were found containing different types of data from varying

147 numbers of participants. Description of the data available from each data resource, and links to 
148 each resource is provided. Moreover, key implications are addressed and underrepresented areas

149 of data are identified.

150

$151 \quad 3$ Methodology

152 Our aim was to conduct the equivalent of a systematic review, which in this paper is

153 described as a 'systematic search'. Therefore, wherever possible, the search for ASD resources

154 closely followed the guidelines and the layout of a systematic literature review, and the

155 methodology followed a rigorous structure and search process. The search strategy was

156 performed by the author, Reem Al-jawahiri. The principal focus of the search was datasets

157 containing data pertaining to symptom severity and symptom profile (e.g. scores on the Autism

158 Diagnostic Observational Schedule (ADOS), or Autism Diagnostic Inventory - Revised (ADI-

159 R), cognitive ability, neuroimaging and biospecimens. We used a number of key-word

160 combinations to identify these resources (see Table 1). While carrying out the search we also

161 identified a number of sources of available genetics and omics data; some of which contained

162 data from participants with ASD, others contained genetic or omics data useful for the study of

163 ASD. Where information about these datasets was readily available on the relevant webpage

164 these data sources have also been included and described here. However, where information

165 about the datasets was less clear, either because it was not readily available via the website, or

166 because it required specialist knowledge to understanding the nature of the material held by the

167 dataset, these resources were not included.

168

1693.1 Search Terms

170 
171 The combinations of search terms used, and the number of results returned from either Scopus or

172 Google for each search term are presented in Table 1.

173

174 Table 1. Search Terms

\begin{tabular}{|c|c|c|}
\hline Keywords & $\begin{array}{l}\text { Database / } \\
\text { Search } \\
\text { Engine }\end{array}$ & $\begin{array}{l}\text { \# of Results on } \\
\text { Primary Search }\end{array}$ \\
\hline $\begin{array}{l}\text { Autism AND (database OR databank OR } \\
\text { repository) }\end{array}$ & Scopus & 1,050 results \\
\hline $\begin{array}{l}\text { Autism AND (database OR databank OR } \\
\text { repository) }\end{array}$ & Google* & $\sim 14,400,000$ results \\
\hline Autism AND data AND sharing & Scopus & 80 results \\
\hline Autism AND data AND sharing & Google* & $\sim 6,310,000$ results \\
\hline $\begin{array}{l}\text { Autism AND (database OR databank OR } \\
\text { repository) AND (EEG OR MEG) }\end{array}$ & Scopus & 19 results \\
\hline $\begin{array}{l}\text { Autism AND (database OR databank OR } \\
\text { repository) AND (EEG OR MEG) }\end{array}$ & Google* & $\sim 515,000$ results \\
\hline $\begin{array}{l}\text { Autism AND (database OR databank OR } \\
\text { repository) AND MRI }\end{array}$ & Scopus & 23 results \\
\hline $\begin{array}{l}\text { Autism AND (database OR databank OR } \\
\text { repository) AND MRI }\end{array}$ & Google* & $\sim 354,000$ results \\
\hline $\begin{array}{l}\text { Autism AND (database OR databank OR } \\
\text { repository) AND (genetic OR genome) }\end{array}$ & Scopus & 315 results \\
\hline $\begin{array}{l}\text { Autism AND (database OR databank OR } \\
\text { repository) AND (genetic OR genome) }\end{array}$ & Google* & $\sim 493,000$ results \\
\hline $\begin{array}{l}\text { Autism AND (database OR databank OR } \\
\text { repository) AND (phenotype OR phenotypic) }\end{array}$ & Scopus & 145 results \\
\hline $\begin{array}{l}\text { Autism AND (database OR databank OR } \\
\text { repository) AND (phenotype OR phenotypic) }\end{array}$ & Google* & $\sim 524,000$ results \\
\hline
\end{tabular}

* Only pages 1 to 3 (inclusive) were assessed. 


\section{$176 \quad 3.2$ Inclusion and Exclusion Criteria}

177 Available data resources that share human ASD data of numerous types including phenotypic,

178 neuroimaging, human brain connectivity matrices, human brain statistical maps, biospecimens,

179 and ASD participant recruitment services were considered eligible for inclusion. Data types,

180 including datasets, databases, databanks, data repositories, institutions offering subject

181 recruitment services, and big-data-initiatives that are currently sharing or have made a public

182 statement of intent to share ASD data at a point in time, were considered eligible regardless of

183 methodology and modality of data collection and analysis. Resources of electronic medical

184 records, clinical trial registries, and data relating to animal studies were excluded. In order to

185 avoid redundant data and clutter, if more than one source housed the same ASD dataset then only

186 the original dataset source or key website was included. Within Scopus, the title, abstract, and

187 keywords of the returned articles were searched using the key search terms. Only articles or

188 webpages written in English were included. The search was carried out between January and

189 March 2016, therefore any new data resources or initiatives made available after March 2016 are

190 not included.

\section{$191 \quad 3.3$ Search Process}

192 The Google search engine and the Scopus electronic database were systematically searched for

193 relevant ASD data resources. The search terms and the different search term combinations used

194 are listed in Table 1. Because Google is a search engine (and not a database with limited results),

195 only pages one to three, inclusive, were assessed; After the third page, the links become less

196 relevant and therefore the limit was set to comprise the first three pages. Naturally, in the case of 
197 Scopus searches, all results were assessed. The results in Scopus are based on searches of the

198 search terms in the title/abstract/keywords of the identified journal articles.

199

200 Relevant metadata articles, bioinformatics articles, and relevant data portals were also

201 identified and were useful to further complete the data search. Some articles described the data

202 resources or datasets, while others presented tables of useful resources for data access and

203 sharing - although not specifically referring to ASD data [i.e. 46-50]. The Neuroimaging

204 Informatics Tools and Resources Clearinghouse, Neuroscience Information Framework, and

205 SciCrunch are among the data portals and registries used in the systematic search for relevant

206 data resources. Further articles / data resources were found through cited reference searching and

207 through the 'related documents' option in the Scopus database.

208

209 The 'information for authors' section of a number of journals, including Nature

210 publishing group journals, Scientific Data journal, PLOS journals, BioMed Central/ GigaScience

211 journal were examined for whether requirements or recommendations were expressed regarding

212 the assimilation of data via supplementary information or via particular data resources /

213 repositories. These journals provided lists of recommended data resources for particular data

214 types for authors wishing to submit their manuscripts and related data. The 'policy' tab of the

215 Wellcome Trust provided a similar, useful list of data resources.

217 Based on titles, potentially relevant articles or webpages were identified and grouped in tabs,

218 before either the abstract or the webpage of all the saved tabs was assessed. Articles and

219 webpages that consisted of items fitting the inclusion criteria were further assessed after 
220 accessing the full text versions and finding the relevant websites of the data resources. Some data

221 providers were contacted for further verification regarding the data type and availability.

222 Duplicates of links leading to the same data resources found from searches, were removed.

223 Similarly, as mentioned earlier, websites hosting the same ASD datasets or overlapping datasets

224 were removed to avoid clutter.

\section{Results}

A total of 33 data resources that provide ASD data were identified. Links to these resources, and a description of the data they hold can be seen in Tables 2 and 3 (in addition to the supporting information in Supplementary File S1). In both tables, the resources are ordered based on two criteria and the first criterion takes precedent over the second. The first criterion is

231 whether they specialize in only ASD data as opposed to also providing data from other populations in addition to the ASD data. For example, for Table 2, since the National Database for Autism Research (NDAR), SFARI, Autism Genetic Resource Exchange (AGRE), Autism Spectrum Database-UK (ASD-UK), and Interactive Autism Network (IAN) all specifically and solely provide ASD data, they are positioned at the top of the list. The second criterion is based on the similarity (among the resources in the respective table) in the general ASD data-type they

237 handle and provide. For example, Autism Brain Imaging Data Exchange (ABIDE) and the Australian EEG Database (AED) both mainly provide neuroimaging ASD data, therefore these two resources are positioned close to each other. 
240 Table 2. A Comprehensive List of ASD Data Resources

\begin{tabular}{|c|c|c|c|c|}
\hline$\overline{\text { URL }}$ & Resource & Data Type Category & Data Type & $\begin{array}{c}\text { Number of Participants with } \\
\text { ASD }\end{array}$ \\
\hline ndar.nih.gov & $\begin{array}{l}\text { National Database for Autism Research } \\
\text { (NDAR)* }\end{array}$ & $\begin{array}{l}\text { Phenotypic, } \\
\text { neuroimaging, } \\
\text { genetic, omics }\end{array}$ & $\begin{array}{l}\text { Phenotypic, neuroimaging, genetic, } \\
\text { omics data }\end{array}$ & $\begin{array}{l}\text { Over } 80,203 \text { participants } \\
\text { (however this number includes } \\
\text { the control participants of the } \\
\text { ASD studies). }\end{array}$ \\
\hline sfari.org & $\begin{array}{l}\text { Simons Foundation Autism Research } \\
\text { Initiative (SFARI)* }\end{array}$ & $\begin{array}{l}\text { Phenotypic, } \\
\text { neuroimaging, } \\
\text { genetic }\end{array}$ & $\begin{array}{l}\text { Phenotypic data, biospecimens, genetic } \\
\text { data, neuroimaging data, participant } \\
\text { recruitment (to recruit SSC families for } \\
\text { additional studies) }\end{array}$ & $\begin{array}{l}\text { Over 3,000 participants (SSC), } \\
\text { over } 200 \text { participants (Simons } \\
\text { VIP), 50,000† participants } \\
\text { (SPARK). }\end{array}$ \\
\hline $\begin{array}{l}\text { research.agre.org/program } \\
\text { /descr.cfm }\end{array}$ & $\begin{array}{l}\text { Autism Genetic Resource Exchange } \\
\text { (AGRE)* }\end{array}$ & $\begin{array}{l}\text { Phenotypic, genetic, } \\
\text { biospecimens }\end{array}$ & $\begin{array}{l}\text { Phenotypic data; genetic data, } \\
\text { biospecimens }\end{array}$ & $\begin{array}{l}\text { Over } 1,700 \text { families with over } \\
\text { 3,300 ASD participants. }\end{array}$ \\
\hline$\frac{\text { iancommunity.org } / \mathrm{cs} / \text { for }}{\text { researchers }}$ & Interactive Autism Network (IAN)* & $\begin{array}{l}\text { ASD participant } \\
\text { recruitment services }\end{array}$ & $\begin{array}{l}\text { Phenotypic data, ASD participant } \\
\text { recruitment services }\end{array}$ & Over 17,000 participants. \\
\hline asd-uk.com & $\begin{array}{l}\text { Autism Spectrum Database-UK (ASD- } \\
\text { UK)* }\end{array}$ & $\begin{array}{l}\text { ASD participant } \\
\text { recruitment services }\end{array}$ & $\begin{array}{l}\text { Phenotypic data, ASD participant } \\
\text { recruitment services }\end{array}$ & Over 3,000 families. \\
\hline autismbrainnet.org & Autism BrainNet & BioBank & $\begin{array}{l}\text { Postmortem brain and related } \\
\text { biospecimens }\end{array}$ & $\begin{array}{l}\text { Over } 25 \text { donations (since } \\
2014) \dagger \text {. }\end{array}$ \\
\hline
\end{tabular}


fcon_1000.projects.nitrc.o rg/indi/abide

$-$

Brainmap.org

neurovault.org

NeuroVault

umcd.humanconnectomep roject.org

datadryad.org

figshare.com

nimhgenetics.org (ABIDE)

BrainMap

$$
\text { Dryad }
$$

FigShare:
Autism Brain Imaging Data Exchange

\section{Australian EEG Database (AED) $\S$}

USC Multimodal Connectivity Database

NIMH Repository and Genomics Resource (NIMH-RGR)
Neuroimaging

Neuroimaging

Human brain statistical maps

Human brain statistical maps

Brain connectivity matrices

General data repository

General data repository

Biospecimens, genetic
Resting state functional magnetic resonance imaging (R-fMRI), structural MRI, phenotypic data

EEG data

fMRI, PET, and structural coordinatebased results $(\mathrm{x}, \mathrm{y}, \mathrm{z})$ in Talairach or MNI space

Unthresholded statistical maps, parcellations, and atlases produced by MRI and PET studies

Brain connectivity matrices of fMRI and DTI

lncRNA, MRI, metabolite, MEG

Phenotypic, statistical, genetic data

Biospecimens (DNA samples and cell lines, Induced Pluripotent Stem Cell (iPSC) and Source Cells), GWAS, genomic sequences
539 participants (ABIDE I), 487 participants (ABIDE II).

50 participants $^{\wedge}$

70 results / articles relevant to ASD functional data (search using BrainMapWeb).

5 studies: $277,60,50,13,218$ participants in each study.

42 (fMRI) participants, 51 (DTI) participants.

4 studies: 2, 34, 12, and 13 participants respectively.

Biospecimens: 4,793 families and 19,359 individuals of which 17,189 have DNA cell lines. Genome-Wide Association Studies (GWAS) Data: 4 studies (1,232 cases, 739 families, 943 families, 935 families). Sequence data (exome): 2,119 cases. 
bristol.ac.uk/alspac/

Avon Longitudinal Study of Parents and Children (ALSPAC)

$\underline{\text { catalog.coriell.org }}$

neurobiobank.nih.gov

kcl.ac.uk/ioppn/depts/ben/

Our-

research/Neurodegenerati

on/brain-bank.aspx Research Resource)

NIH NeuroBioBank (NBB)

Medical Research Council London Neurodegenerative Diseases Brain Bank
Phenotypic, clinical, Phenotypic, clinical, biospecimens, biospecimens, genetic genetic (including GWAS, SNPs,

Coriell BioRepositories (including Autism
BioBank

BioBank

BioBank
Postmortem brain and spinal cord tissue
VNTRs, in addition to sequence data from UK10K project available via EGA), ALPAC data linked with data (e.g. routine health and social records) from external sources, bespoke data||

96 participants (as identified via follow up questionnaires completed by carers for when the proband was 9 years old)

Cell cultures, DNA samples, and induced pluripotent stem cells

Postmortem brain and related biospecimens

64 ASD cases. 22 ASD

suspected.

4 ASD cases.
158 ASD cases

$241 *$ Data-type is described in more detail in Supplementary File S1.

$242 \uparrow$ The data is not yet available: It is intended to be available in a future date according to the SFARI website.

$243 \S$ There is no website or portal for the AED resource; however, the data is available via email requests to aed@newcastle.edu.au

$244 \wedge$ The approximate number of ASD participants was found via email correspondence with aed@newcastle.edu.au

$245 \ddagger$ Accurate information regarding the approximate number of participants with ASD is not readily available on the website, due to the nature of the search 246 functionality.

247 Data specifically from ASD participants are not necessarily available in all the different data types described in this table (therefore further specific enquiries

248 directed to the ALSPAC team is advised). 
250 Table 3. Genetics and Omics Data Resources Either from Individuals with ASD or Containing Data Relevant to the Study of ASD*

\begin{tabular}{|c|c|c|c|c|}
\hline URL & Resource* & $\begin{array}{l}\text { Data Type } \\
\text { Category }\end{array}$ & Data Type & Notes \\
\hline mss.ng & MSSNG & $\begin{array}{l}\text { Genetic/ } \\
\text { Genomic }\end{array}$ & $\begin{array}{l}\text { Phenotypic, genomic (whole } \\
\text { genome sequencing of blood } \\
\text { DNA) }\end{array}$ & $\begin{array}{l}10,000 \text { participants. However, data from } \\
\text { only } 3000 \text { probands is currently available. }\end{array}$ \\
\hline gene.sfari.org & $\begin{array}{l}\text { Simons Foundation Autism Research } \\
\text { Initiative Gene (SFARI Gene) }\end{array}$ & Gene Catalogue & $\begin{array}{l}\text { Animal Model, Protein } \\
\text { Interaction (PIN), Gene } \\
\text { Scoring, CNV }\end{array}$ & $\begin{array}{l}\text { An up-to-date, manually annotated } \\
\text { reference set of ASD-linked genes. }\end{array}$ \\
\hline projects.tcag.ca/autism & $\begin{array}{l}\text { Autism Chromosome Rearrangement } \\
\text { Database (ACRD) }\end{array}$ & Gene Catalogue & $\begin{array}{l}\text { Genomic structural variation } \\
\text { data - CNVs }\end{array}$ & $\begin{array}{l}\text { A curated catalogue of structural variation } \\
\text { related to ASD extracted from publicly } \\
\text { available literature and unpublished data. }\end{array}$ \\
\hline $\begin{array}{l}\text { autismkb.cbi.pku.edu.cn/inde } \\
\text { x.php }\end{array}$ & Autism Knowledgebase (AutismKB) & Gene Catalogue & $\begin{array}{l}\text { A collection of genes and } \\
\text { variations associated with ASD } \\
\text { with annotations }\end{array}$ & - \\
\hline$\frac{\text { ncbi.nlm.nih.gov/guide/sitema }}{\mathrm{p} /}$ & $\begin{array}{l}\text { National Center for Biotechnology } \\
\text { Information (NCBI) }\end{array}$ & Genetics, omics & $\begin{array}{l}\text { A collection of multiple } \\
\text { resources - omics and } \\
\text { sequencing data }\end{array}$ & - \\
\hline$\underline{\text { ebi.ac.uk/services/all }}$ & $\begin{array}{l}\text { European Molecular Biology Laboratory } \\
\text { (EMBL-EBI) }\end{array}$ & Genetics, omics & $\begin{array}{l}\text { A collection of multiple } \\
\text { resources - omics and } \\
\text { sequencing data }\end{array}$ & - \\
\hline uniprot.org & Universal Protein Resource (UniProt) & $\begin{array}{l}\text { Protein } \\
\text { sequences }\end{array}$ & $\begin{array}{l}\text { Protein sequences and their } \\
\text { annotations }\end{array}$ & $\begin{array}{l}\text { Can be found among EMBL-EBI resources. } \\
91 \text { (reviewed) and } 346 \text { (unreviewed) } \\
\text { protein records associated with ASD. }\end{array}$ \\
\hline ebi.ac.uk/ega & $\begin{array}{l}\text { The European Genome-phenome } \\
\text { Archive (EGA) }\end{array}$ & $\begin{array}{l}\text { Omics - } \\
\text { Functional } \\
\text { genomics }\end{array}$ & $\begin{array}{l}\text { Interaction of genotype and } \\
\text { phenotype (including data from } \\
\text { UK10K project) }\end{array}$ & Can be found among EMBL-EBI resources. \\
\hline
\end{tabular}


thebiogrid.org

gpmdb.thegpm.org/index.html Global Proteome Machine Database (GPM DB)

peptideatlas.org

PeptideAtlas

ddbj.nig.ac.jp/index-e.html

DNA DataBank of Japan (DDBJ)

$\underline{\operatorname{chr} 7 . o r g}$

mirbase.org

gbrowse.csbio.unc.edu/cgi$\underline{\text { bin/gb2/gbrowse/slep }}$

Biological General Repository for Interaction Datasets (BioGRID)
Omics

Omics -

Proteomics

Proteomics data from tandem mass spectrometry

Omics -

Proteomics

Peptide sequences, mapping proteome information/data

DNA and RNA DNA and RNA sequences sequences

DNA sequences

DNA sequence and annotation of the entire human chromosome 7

miRNA

sequences

Sullivan Lab Evidence Project (SLEP)

Genetics, omics

miRNA sequences and annotation

A collection of genes and variations associated with ASD with annotations
Resource that archives and disseminates genetic and protein interaction data.

Open-source system for analyzing, storing, and validating proteomics information derived from tandem mass spectrometry.

A collection of peptides identified in a large set of tandem mass spectrometry proteomics experiments.

Annotated collection of all publicly available nucleotide sequences and their translated amino acid sequences.

84 cases.

Findings from genome wide linkage

(GWL), genome wide association (GWA), and microarray (MA) studies for ASD.

* The resources listed in this table contain data either from individuals with ASD or data relevant to ASD research that is collected from non-affected individuals

252 (e.g. from individuals with certain genetic profiles or syndromes related to ASD research). 
The 33 identified resources are presented in two separate tables (18 resources in Table 2 and

25415 resources in Table 3) based broadly on the category of data type that the resources hold. The

255 resources in Table 2 contain various types of data including phenotypic data, neuroimaging data,

256 biospecimens, brain connectivity matrices, and brain statistical maps. The table also includes

257 data providers that offer ASD participant recruitment services (i.e. ASD-UK and IAN). Four

258 further resources (i.e. Neuroimaging Informatics Tools and Resources Clearinghouse - Image

259 Repository, the LONI Image and Data Archive, COINS Data Exchange, Neuroinformatics

260 Database, Longitudinal Online Research and Imaging System) were identified but were omitted

261 due to them providing overlapping ASD datasets from the same source: ABIDE datasets.

262 Therefore, only the ABIDE website (fcon_1000.projects.nitrc.org/indi/abide) is included in

263 Table 2.

264

265 As can be seen in Table 2, data size and participant numbers vary among the resources and

266 data modalities. Moreover, certain resources provide data from large scale studies such as data

267 from SFARI and ABIDE, where all participants take part in the same protocol, whereas other

268 resources such as NDAR and Dryad provide data from very small studies, e.g. studies containing

269 data from 2, 12, 13 and 34 participants with ASD. Additionally, some resources are based on

270 initiatives and collaborations that aimed to collect primary data (not from prior studies) for the

271 purpose of sharing it to the scientific community. For example, AED is a resource with EEG data

272 from over 20,000 patient records (however only 50 ASD EEG records) made available through

273 the collaboration between John Hunter Hospital and the University of Newcastle, Australia. 
275 The data types of the resources in Table 3 are somewhat more homogenous and would fall 276 under the categories of genomic, genetic, and omics / proteomics data. For example, data types

277 include gene catalogue (e.g. CNVs and protein interactions), sequencing data (e.g. DNA

278 sequencing, RNA sequencing, and protein sequencing), functional genomics data (interaction of

279 genotype and phenotype), genetic and protein interaction data, in addition to other genetic and

280 omics data. The relevant data from these resources are either genetic or omics data shown to be

281 potentially involved with ASD (collected from unaffected participants) or data from participants

282 with ASD. Several resources, namely, NDAR, SFARI, AGRE, and NIMH Repository and

283 Genomics Resource, also provide various genetic data, however these resources are grouped with

284 the resources in Table 2 because they also provide additional data-types such as phenotypic,

285 neuroimaging, and / or biospecimens.

286

287

Some of the resources in Tables 2 and 3 such as NDAR, SFARI, AGRE, and MSSNG, are 288 specialized in ASD data and aim to share only autism-related data. While other resources, due to the purpose of the resource or the nature of the data, aim to share data involved with numerous populations and conditions. Nevertheless, these resources also housed data involved with ASD research. Examples of non-ASD specialized resources include Coriell BioRepositories and

292 Universal Protein Resources. The resources vary in the way the data is described or made 293 viewable, searchable, and accessible for researchers. For example, different informatics portals 294 or systems (see the 'URL' columns in Tables 2 and 3, and / or the metadata articles in File S1) 295 are used to achieve the task of data sharing. 296 
NDAR, SFARI, AGRE, MSSNG, IAN, and ASD-UK are among the key resources which 298 provide ASD data. NDAR [51] is the largest ASD resource housing ASD data of various types from numerous sources. It is a US NIH-funded initiative that aims to "accelerate progress in

300 ASD research through data sharing, data harmonization, and the reporting of research results"

301 (ndar.nih.gov). NDAR shares various types of data: phenotypic data (e.g. clinical and diagnostic

302 assessments such as the Autism Diagnostic Inventory (ADI) and ADOS); neuroimaging data

303 (e.g. MEG, fMRI, and DTI); genetic and omics data (e.g. DNA (Microarray and Sequencing) and

304 RNA (Microarray and Sequencing)) related to human participants. NDAR is evolving and it is

305 now incorporated under the NIMH Data Archive (data-archive.nimh.nih.gov). Researchers can

306 access the data without any fees, however they need to first request and gain approval for access

307 [see 52, for a flow diagram for data access]. The NDAR system enables data to be aggregated

308 not only from other major data resources (e.g. SFARI, AGRE, and IAN), but also from qualified

309 autism researchers regardless of funding source. Currently, NDAR contains data from over

31080,000 participants (however this number includes neurotypical control participants of the ASD

311 studies).

312

313 SFARI is an ASD data resource that shares very well-characterized participant data due to

314 the rigorous standardization and coordination among the SFARI-funded investigators (currently

315 over 250 investigators). SFARI's aim is to "improve the understanding, diagnosis and treatment

316 of autism spectrum disorders by funding innovative research of the highest quality and

317 relevance" (sfari.org/about-sfari) . SFARI also aims "to facilitate and drive research in the field as

318 a whole" through granting data access to autism researchers (regardless of their funding source).

319 Approved researchers are granted access to the data without a fee, however biospecimen data is 
320 available on a modest fee-for use basis. SFARI's data are segmented and collected from three

321 large cohorts: Simons Simplex Collection (SSC), Simons Variation in Individuals Project

322 (Simons VIP), and Simons Foundation Powering Autism Research for Knowledge (SPARK).

323 The SSC is intended to be especially useful for investigating de novo (new) mutations due to the

324 cohort comprising of nearly 3,000 simplex families. Simons VIP contains data from over 200

325 participants with recurrent genetic variants involved with ASD (i.e. deletion and duplication of

326 chromosomal region 16p11.2 and 1q21.1). SPARK is a new initiative involving collecting

327 phenotypic and biospecimen data from 50,000 participants with ASD. This initiative will later

328 provide researchers with participant recruitment services through retaining a database of the

329 SPARK participants who consent to be re-contacted for future studies.

331 AGRE is a resource that is mainly supported by Autism Speaks and is among the leading

332 resources committed to advancing ASD genetic research. AGRE shares phenotypic (e.g. ADI-R

333 and ADOS), genetic (e.g. high-density SNP and Genome Wide Association Study data), and

334 biospecimen data (e.g. cell lines, DNA and plasma) of over 1,700 well defined multiplex and

335 simplex families with over 3,300 participants with ASD. AGRE can also provide participant

336 recruitment services. In order to access data, researchers need to request data and pay per sample

337 fee for obtaining biospecimen data.

338

MSSNG is the largest ASD genomic resource made available to the research community. It

340 is the outcome of the diverse collaboration efforts between Autism Speaks, SickKids Hospital,

341 University of Toronto, and Google. MSSNG's aim is "to provide the best resources to enable the

342 identification of many subtypes of autism, which may lead to better diagnostics, as well as 
343 personalized and more accurate treatments" (research.mss.ng). The target is to sequence and

344 share data from whole genome sequencing of blood DNA of 10,000 families affected by ASD.

345 The cohorts consist of AGRE participants, but could later include other well-phenotyped cohorts.

346 Over 5000 whole genome sequences (and coded phenotype data) with nearly 3000 sequences

347 from participants with ASD are freely available to approved researchers.

349 IAN and ASD-UK are both useful resources based in the US and the UK respectively, that 350 enable the collaboration between researchers and participants. With the aim of facilitating and

351 advancing ASD research, these resources provide approved researchers with participant

352 recruitment services and phenotypic data. The data that can be accessed consist of certain

353 phenotypic data (e.g. Social Communication Questionnaire) along with medical data (e.g.

354 medical history). Researchers can request and access these resources at a cost. To date, IAN and

355 ASD-UK registered over 17,000 participants with ASD and over 3,000 families, respectively.

356 For more information on NDAR, SFARI, AGRE, ASD-UK, and IAN in addition to a list of

357 relevant metadata articles, see Supplementary File S1.

\section{Discussion}

The systematic search showed that there are at least 33 data resources and initiatives that

361 aim to share available ASD data to interested researchers, and therefore further advance ASD

362 research and understanding of its heterogeneity and potential subtypes. These resources and

363 initiatives have undoubtedly had, and will continue to exert, significant influence on the field of

364 ASD research. Particularly, notable initiatives such as NDAR and SFARI can, arguably, have an 
365 impact on the nature of ASD research and the process of data sharing. Although NDAR and

366 SFARI share similar aims, they differ in their approach and thus affect the field differently. Both

367 resources aim for the advancement of ASD research through supporting certain ASD projects

368 and facilitating the secondary analysis of data. Additionally, NDAR and SFARI are specialized

369 ASD resources that aim to accommodate for ASD-specific data types and methodologies. As a

370 result, these resources led to new findings and numerous publications in the field. Each resource,

371 however, accomplishes its aims through a distinctive approach and data sharing process, which

372 arguably differentially influences the nature of ASD research and data sharing process.

373 Specifically, each resource adopted a different approach to obtaining its primary data. Even

374 though NDAR and SFARI initially obtained and shared data collected solely from particular

375 projects that they funded, NDAR currently obtains its primary data from multiple sources

376 including over $120 \mathrm{NIH}$-funded investigators in addition to other autism researchers irrespective

377 of funding source. SFARI, on the other hand, obtains its primary data only from SFARI-funded

378 investigators mainly involved in large projects. One approach (i.e. NDAR's approach) interacts

379 with various sources that range in the scale of their data, while the other interacts with sources

380 that offer large datasets. Consequently, the former approach supports the secondary analysis and

381 sharing of smaller scale data, while the latter drives the reuse of large datasets and the sharing of

382 large data.

383

384 Both approaches have their merits and there are advantages and disadvantages of the sharing

385 of either small or big data. One advantage of sharing small data is to allow the scientific

386 community to make use of the rich variety of research data that is collected by small independent

387 research efforts. As ASD is a clinical population that is typically challenging to recruit and 
388

389

390

391

392

393

394

395

396

397

398

399

400

401

402

403

404

405

406

407

408

409

410

examine, especially in its more severe form, most ASD studies therefore are comprised of small sample sizes or small data. Collectively, these small efforts would make up a large sum of studies, with data from various specialties and modalities. This can be valuable for secondary analysis if the data is shared, especially if the datasets can either be analysed individually or integrated into different combinations. Whether to augment the scale of the dataset or to explore links between data from different modalities and levels, integrating datasets from different studies is a potentially greatly beneficial yet challenging task that some data resources are tackling. A model initiative and collaboration between NDAR and SFARI towards this direction resulted in the development of a tool that ultimately helps in identifying overlapping data and in linking data from different studies. The tool is a free software application that assigns global unique identifiers (GUID) to research participants [see 53-55]. Using an algorithm derived from four input variables taken from a participant's birth certificate, the GUID tool produces a sequence of letters and numbers to serve as a standardized, unique, and anonymized code specific to a research participant, regardless of the study the participant is engaged in. The generated GUID will always be the same for the particular participant and thus will be used across studies if the participant participates in more than one study. This system would make it possible to trace data across studies of the same participant and would facilitate data overlap detection, especially if more studies and data resources use GUIDs. Hence, NDAR requires all prospective studies to include GUIDs in the data submission plan (and encourages retrospective studies to include GUIDs or pseudo-GUIDs). Other than NDAR and SFARI, a number of major ASD resources, namely, IAN, AGRE, and Autism BrainNet generate GUIDs for their studies making GUIDs an even more effective tool. 
411 However, despite these efforts, combining or linking small different datasets remains a

412 difficult task requiring sophisticated integration tools and the participation and collaboration of

413 data resources. While in the case of big data, the datasets offered are large homogenous datasets

414 that although could also benefit from being combined with other datasets, it is in itself ready for

415 re-analysis with great statistical power. Despite the challenge of integrating small data, sharing

416 data (in both cases small or big) would encourage more transparency and rigorous research,

417 whether because the re-analysis would uncover limitations or errors not previously identified; or

418 because the prospect of sharing data, encourages the respective researcher to be more cautious in

419 their analysis and/or reporting. This can be implied from the findings of a recent report, which

420 found that psychology studies that were unwilling to share data were associated with weaker

421 statistical evidence, and more errors in statistical reporting of results in comparison to studies

422 with shared data [56].

423

424 Other than the impact of the available ASD resources, a second point to consider is the role

425 of retrospective data and whether it has a role to play towards the shift to big data and open data.

426 Retrospective data here is referred to data that is previously collected by researchers, clinicians,

427 or other entities for the purpose of the respective studies or for keeping medical records and / or

428 other information about patients or groups of interest. Naturally, there is a large sum of ASD

429 retrospective data whether cumulatively from small independent studies or from clinical sources

430 with many records and information. Although invaluable for the advancement of ASD research

431 and for medical purposes, this type of data is collected without taking into account the prospect

432 of sharing it. If shared, this ASD data can be used by researchers for secondary or primary

433 analysis, respectively. However, the sharing of data that is not initially prepared for the purpose 
434 of sharing can be challenging and the available data-sharing resources lack initiatives that

435 address this challenge. The resources should tailor to retrospective data and aim to integrate and

436 present annotated ASD data that later gained permissions for sharing. NDAR along with some

437 EEG-specialized and general-purpose resources, such as AED, Dryad, and FigShare, among

438 others, allow for the sharing of certain retrospective data, however tailored resources that cater to

439 retrospective data from various sources and modalities is still limited.

A third point to consider is the difficulty of finding certain ASD data and sufficient data

442 annotation that are of interest to researchers. As shown in Tables 2, 3, and the Supplementary

443 File S1, data from certain modalities are underrepresented or even lacking. For example, none of

444 the available resources provide access to eye-tracking data obtained from participants with ASD.

445 In addition, very few resources provide EEG or MEG data obtained from participants with ASD:

446 despite the large number of datasets being held by NDAR, no EEG datasets and only 21 MEG

447 datasets are available from individuals with ASD. Even when this type of data (or neuroimaging

448 data generally) is found, researchers face other difficulties in finding certain information about

449 the data. One bit of data information that is essential to researchers is pertaining to the level of

450 data quality (both the raw and processed data) in accordance with the standards of the specific

451 field and modality type. However, a system that describes quality is not developed and the data-

452 sharing process, typically, does not permit researchers to have a quick view of the actual data. If

453 data can be viewed (before it is officially obtained), researchers would be better able to

454 determine the quality and feasibility of the data in relation to the researchers' interests, skills, and

455 resources. The available ASD data websites either describe or display the available data (or

456 both), however it can be challenging at times to find specific information relating to sample size 
457 and other details about the data. For example, it is unclear in certain cases whether the mentioned

458 sample size, relating to an available dataset, is that of solely participants with ASD or whether it

459 is inclusive of control participants. Furthermore, information about the data that will be shared

460 from on-going studies is very limited if at all reported. Generally, although the current data-

461 websites describe or annotate the data in a certain way, further comprehensive and clear

462 annotation is necessary along with a better inclusion of data from different modalities.

463

\section{Concluding Remarks}

Here, we have identified 33 resources that provide data obtained from individuals with ASD to the research community. While we are confident that we have identified the vast majority, if not all, of the publically available data sources that provide behavioural and neuroimaging data from individuals with ASD, we may not have captured some resources in the cases where research groups have data that they are willing to share, but did not publicize the data in a way that was identifiable via our key-word search. The resources and data-websites are not static:

471 they are improving and the number of available data is increasing in size and variety. Notably,

472 the drive for big data, sharing data, and for accelerating research on complex heterogeneous

473 disorders such as autism is not only leading to the on-going growth of current initiatives, but also 474 of the development of new ones, e.g. MSSNG.

\section{$475 \quad 7$ References}

1. Focus on big data. Nat Neurosci. 2014 Nov;17(11):1429. 
477

478

479

480

481

482

483

484

485

486

487

488

489

490

491

492

493

494

495

496

497

498

499

500

501

502

503

504

505

506

507

2. Eickhoff S, Nichols TE, Van Horn JD, Turner JA. Sharing the wealth: neuroimaging data repositories. Neuroimage. 2016;124:1065-1068.

3. Oliver, J. M., Slashinski, M. J., Wang, T., Kelly, P. A., Hilsenbeck, S. G., \& McGuire, A. L. (2012). Balancing the risks and benefits of genomic data sharing: genome research participants' perspectives. Public Health Genomics, 15(2), 106-114.

doi:10.1159/000334718

4. Cummings, J. A., Zagrodney, J. M., \& Day, T. E. (2015). Impact of Open Data Policies on Consent to Participate in Human Subjects Research: Discrepancies between Participant Action and Reported Concerns. PLoS One, 10(5), e0125208. doi:10.1371/journal.pone.0125208

5. Geschwind DH, Levitt P. Autism spectrum disorders: developmental disconnection syndromes. Curr Opin Neurobiol. 2007 2;17(1):103-111.

6. American PA, American Psychiatric Association Task Force oDSM, American Psychiatric Association DSMIVTF, Apa, A.P.A, Asociación Psiquiátrica A, Asociación Americana dP, Joint Information Service of the American Psychiatric Association and the National Association for MH, American Medico-Psychological A, and American Psychiatric Institute for Research aE. Diagnostic and statistical manual of mental disorders DSM-5. 5th ed. ed. Arlington, VA: Arlington, VA American Psychiatric Association, c2013; 2013.

7. Hewitson L. Scientific challenges in developing biological markers for autism. OA Autism. 2013;1(1):7.

8. Happé F, Ronald A, Plomin R. Time to give up on a single explanation for autism. Nat Neurosci. 2006;9(10):1218-1220.

9. Fountain C, Winter AS, Bearman PS. Six developmental trajectories characterize children with autism. Pediatrics. 2012;129(5).

10. Gotham K, Pickles A, Lord C. Trajectories of autism severity in children using standardized ADOS scores. Pediatrics. 2012;130(5):e1278.

11. Venker CE, Ray-Subramanian C, Bolt DM, Weismer SE. Trajectories of autism severity in early childhood. J Autism Dev Disord. 2014;44(3):546-563.

12. Werling DM, Geschwind DH. Sex differences in autism spectrum disorders. Curr Opin Neurol 2013 Apr;26(2):146-153. 
508

509

510

511

512

513

514

515

516

517

518

519

520

521

522

523

524

525

526

527

528

529

530

531

532

533

534

535

536

13. Sebat J, Lakshmi B, Malhotra D, Troge J, Lese-Martin C, Walsh T, Yamrom B, Yoon S, Krasnitz A, Kendall J, Leotta A, Pai D, Zhang R, Lee Y-H, Hicks J, Spence SJ, Lee AT, Puura K, Lehtimäki T, Ledbetter D, Gregersen PK, Bregman J, Sutcliffe JS, Jobanputra V, Chung W, Warburton D, King M-C, Skuse D, Geschwind DH, Gilliam TC, Ye K, and Wigler M. Strong association of de novo copy number mutations with autism. Science. 2007;316(5823):445.

14. Marshall CR, Noor A, Vincent JB, Lionel AC, Feuk L, Skaug J, Shago M, Moessner R, Pinto D, Ren Y, Thiruvahindrapduram B, Fiebig A, Schreiber S, Friedman J, Ketelaars CEJ, Vos YJ, Ficicioglu C, Kirkpatrick S, Nicolson R, Sloman L, Summers A, Gibbons CA, Teebi A, Chitayat D, Weksberg R, Thompson A, Vardy C, Crosbie V, Luscombe S, Baatjes R, Zwaigenbaum L, Roberts W, Fernandez B, Szatmari P, and Scherer SW. Structural variation of chromosomes in autism spectrum disorder. Am J Hum Genet. 2008;82(2):477-488.

15. Banerjee-Basu S, Packer A. SFARI Gene: an evolving database for the autism research community. Dis Model Mech. 2010;3(3-4):133-135.

16. Cholemkery H, Medda J, Lempp T, Freitag CM. Classifying Autism Spectrum Disorders by ADI-R: Subtypes or Severity Gradient? Journal of autism and developmental disorders 2016;46(7):2327-2339.

17. Georgiades S, Szatmari P, Boyle M, Hanna S, Duku E, Zwaigenbaum L, Bryson S, Fombonne E, Volden J, Mirenda P, Smith I, Roberts W, Vaillancourt T, Waddell C, Bennett $\mathrm{T}$, and Thompson A. Investigating phenotypic heterogeneity in children with autism spectrum disorder: a factor mixture modeling approach. J Child Psychol Psychiatry. 2013;54(2):206-215.

18. Doshi-Velez F, Ge Y, Kohane I. Comorbidity clusters in autism spectrum disorders: an electronic health record time- series analysis. Pediatrics. 2014;133(1):e54.

19. Liu X-Q, Georgiades S, Duku E, Thompson A, Devlin B, Cook EH, Wijsman EM, Paterson AD, and Szatmari P. Identification of Genetic Loci Underlying the Phenotypic Constructs of Autism Spectrum Disorders. Journal of the American Academy of Child and Adolescent Psychiatry 2011;50(7):687-696.e13. 
537

538

539

540

541

542

543

544

545

546

547

548

549

550

551

552

553

554

555

556

557

558

559

560

561

562

563

564

565

566

567

20. Veatch OJ, Veenstra-vanderweele J, Potter M, Pericak-vance MA, Haines JL. Genetically meaningful phenotypic subgroups in autism spectrum disorders. Genes, Brain and Behavior. 2014;13(3):276-285.

21. Vieland V, Hallmayer J, Huang Y, Pagnamenta A, Pinto D, Khan H, Monaco A, Paterson A, Scherer S, Sutcliffe J, and Szatmari P. Novel method for combined linkage and genome- wide association analysis finds evidence of distinct genetic architecture for two subtypes of autism. J Neurodevelop Disord. 2011;3(2):113-123.

22. Wiggins LD, Robins DL, Adamson LB, Bakeman R, Henrich CC. Support for a dimensional view of autism spectrum disorders in toddlers. J Autism Dev Disord. 2012;42(2):191-200.

23. Kim SH, Macari S, Koller J, Chawarska K. Examining the phenotypic heterogeneity of early autism spectrum disorder: subtypes and short- term outcomes. J Child Psychol Psychiatry. 2016;57(1):93-102.

24. Hu, V. W., \& Steinberg, M. E. (2009). Novel clustering of items from the Autism Diagnostic Interview-Revised to define phenotypes within autism spectrum disorders. Autism Res, 2(2), 67-77. doi:10.1002/aur.72

25. Greaves-Lord K, Eussen M, Verhulst F, Minderaa R, Mandy W, Hudziak J, Steenhuis M, Nijs P, and Hartman C. Empirically based phenotypic profiles of children with pervasive developmental disorders: interpretation in the light of the DSM- 5. J Autism Dev Disord. 2013;43(8):1784-1797.

26. Lai, M. C., Lombardo, M. V., Chakrabarti, B., \& Baron-Cohen, S. (2013). Subgrouping the autism "spectrum": reflections on DSM-5. PLoS Biol, 11(4), e1001544. doi:10.1371/journal.pbio.1001544

27. Hus V, Pickles A, Cook EH, Risi S, Lord C. Using the Autism Diagnostic InterviewRevised to increase phenotypic homogeneity in genetic studies of autism. Biol Psychiatry. 2007;61(4):438-448.

28. Bishop S, Hus V, Duncan A, Huerta M, Gotham K, Pickles A, Kreiger A, Buja A, Lund $\mathrm{S}$, and Lord C. Subcategories of restricted and repetitive behaviors in children with autism spectrum disorders. J Autism Dev Disord. 2013;43(6):1287-1297.

29. Liu X, Paterson AD, Szatmari P. Genome-wide linkage analyses of quantitative and categorical autism subphenotypes. Biol Psychiatry. 2008;64(7):561-570. 
568

569

570

571

572

573

574

575

576

577

578

579

580

581

582

583

584

585

586

587

588

589

590

591

592

593

594

595

596

30. Ausderau KK, Furlong M, Sideris J, Bulluck J, Little LM, Watson LR, Boyd BA, Belger A, Dickie VA, and Baranek GT. Sensory subtypes in children with autism spectrum disorder: latent profile transition analysis using a national survey of sensory features. J Child Psychol Psychiatry. 2014;55(8):935-944.

31. Uljarević M, Lane A, Kelly A, Leekam S. Sensory subtypes and anxiety in older children and adolescents with autism spectrum disorder. Autism Research 2016:n/a-n/a.

32. Buxbaum JD, Silverman JM, Smith CJ, Kilifarski M, Reichert J, Hollander E, Lawlor BA, Fitzgerald M, Greenberg DA, and Davis KL. Evidence for a susceptibility gene for autism on chromosome 2 and for genetic heterogeneity. The American Journal of Human Genetics 2001;68(6):1514-1520.

33. Shao Y, Cuccaro ML, Hauser ER, Raiford KL, Menold MM, Wolpert CM, Ravan SA, Elston L, Decena K, Donnelly SL, Abramson RK, Wright HH, Delong GR, Gilbert JR, and Pericak-Vance M. Fine Mapping of Autistic Disorder to Chromosome 15q11- q13 by Use of Phenotypic Subtypes. Am J Hum Genet. 2003;72(3):539-548.

34. Chaste P, Klei L, Sanders SJ, Hus V, Murtha MT, Lowe JK, Willsey AJ, Moreno-DeLuca D, Yu TW, Fombonne E, Geschwind D, Grice DE, Ledbetter DH, Mane SM, Martin DM, Morrow EM, Walsh CA, Sutcliffe JS, Lese Martin C, Beaudet AL, Lord C, State MW, Cook EH, and Devlin B. A genome- wide association study of autism using the Simons simplex collection: does reducing phenotypic heterogeneity in autism increase genetic homogeneity? Biol Psychiatry. 2015;77(9):775-784.

35. Stessman, H. A., Bernier, R., \& Eichler, E. E. (2014). A genotype-first approach to defining the subtypes of a complex disease. Cell, 156(5), 872-877. doi:10.1016/j.cell.2014.02.002

36. Stessman, H. A., Turner, T. N., \& Eichler, E. E. (2016). Molecular subtyping and improved treatment of neurodevelopmental disease. Genome Med, 8(1), 22. doi:10.1186/s13073-016-0278-z

37. Publications resulting from the Simons VIP [Available from: https://sfari.org/resources/autism-cohorts/simons-vip/publications-resulting-from-thesimons-vip]. 
597

598

599

600

601

602

603

604

605

606

607

608

609

610

611

612

613

614

615

616

617

618

619

620

621

622

623

624

625

626

627

38. Consortium SV. Simons Variation in Individuals Project (Simons VIP): a genetics-first approach to studying autism spectrum and related neurodevelopmental disorders. Neuron. 2012;73(6):1063-7.

39. Hanson E, Bernier R, Porche K, Jackson FI, Goin-Kochel RP, Snyder LG, Snow AV, Wallace AS, Campe KL, Zhang Y, Chen Q, D’Angelo D, Moreno-De-Luca A, Orr PT, Boomer KB, Evans DW, Kanne S, Berry L, Miller FK, and Olson J. The Cognitive and Behavioral Phenotype of the 16p11.2 Deletion in a Clinically Ascertained Population. Biological psychiatry. 2015;77(9):785-93.

40. Steinman KJ, Spence SJ, Ramocki MB, Proud MB, Kessler SK, Marco EJ, Green Snyder L, Angelo D, Chen Q, Chung WK, and Sherr EH. 16p11.2 deletion and duplication: Characterizing neurologic phenotypes in a large clinically ascertained cohort. American Journal of Medical Genetics Part A. 2016;170(11):2943-55.

41. Jenkins J, Chow V, Blaskey L, Kuschner E, Qasmieh S, Gaetz L, Edgar JC, Mukherjee P, Buckner R, Nagarajan SS, Chung WK, Spiro JE, Sherr EH, Berman JI, and Roberts TPL. Auditory Evoked M100 Response Latency is Delayed in Children with 16p11.2 Deletion but not 16p11.2 Duplication. Cerebral Cortex. 2016;26(5):1957-64.

42. Leblanc J, Nelson C. Deletion and duplication of 16p11.2 are associated with opposing effects on visual evoked potential amplitude. Molecular Autism. 2016;7.

43. Qureshi AY, Mueller S, Snyder AZ, Mukherjee P, Berman JI, Roberts TPL, Nagarajan SS, Spiro JE, Chung WK, Sherr EH, and Buckner RL. Opposing brain differences in 16 p11.2 deletion and duplication carriers. The Journal of neuroscience : the official journal of the Society for Neuroscience. 2014;34(34):11199.

44. Chang YS, Owen JP, Pojman NJ, Thieu T, Bukshpun P, Wakahiro MLJ, Marco EJ, Berman JI, Spiro JE, Chung WK, Buckner RL, Roberts TPL, Nagarajan SS, Sherr EH, and Mukherjee P. Reciprocal white matter alterations due to $16 \mathrm{p} 11.2$ chromosomal deletions versus duplications. Human Brain Mapping. 2016;37(8):2833-48.

45. Hudac CM, Kresse A, Aaronson B, Deschamps TD, Webb SJ, Bernier RA. Modulation of mu attenuation to social stimuli in children and adults with $16 \mathrm{p} 11.2$ deletions and duplications. Journal of neurodevelopmental disorders. 2015;7(1):25.

46. Van Horn JD, Scott TG, Rockmore D, Michael SG. Sharing neuroimaging studies of human cognition. Nat Neurosci. 2004;7(5):473. 
628

629

630

631

632

633

634

635

636

637

638

639

640

641

642

643

644

645

646

647

648

649

650

651

652

653

\section{$654 \quad 8$ Supporting Information}

655

656 Supplemental File S1. Metadata of ASD Resources and Detailed Information on Key ASD 657 Resources.

47. Van Horn JD, Wolfe J, Agnoli A, Woodward J, Schmitt M, Dobson J, Schumacher S, and Vance B. Neuroimaging databases as a resource for scientific discovery. Int Rev Neurobiol. 2005;66:55-87.

48. Van Horn JD, Toga AW. Is it time to re- prioritize neuroimaging databases and digital repositories? Neuroimage. 2009;47(4):1720-1734.

49. Ferguson AR, Nielson JL, Cragin MH, Bandrowski AE, Martone ME. Big data from small data: data- sharing in the ' long tail' of neuroscience. Nat Neurosci. 2014;17(11):1442-1447.

50. Poldrack R, Gorgolewski K. Making big data open: data sharing in neuroimaging. Nat Neurosci. 2014;17(11):1510-1517.

51. Hall D, Huerta M, McAuliffe M, Farber G. Sharing heterogeneous data: the national database for autism research. Neuroinform. 2012;10(4):331-339.

52. Payakachat N, Tilford J, Ungar W. National Database for Autism Research (NDAR): big data opportunities for health services research and health technology assessment. Pharmacoeconomics. 2016;34(2):127-138.

53. Johnson SB, Whitney G, McAuliffe M, Wang H, McCreedy E, Rozenblit L, and Evans CC. Using global unique identifiers to link autism collections. Journal of the American Medical Informatics Association. 2010;17(6):689-95.

54. GUID Tool [Available from: https://ndar.nih.gov/tools_guid_tool.html].

55. Rudacille D. Researchers debut unique identifiers for study participants 2010. Available from: https://spectrumnews.org/news/researchers-debut-unique-identifiers-for-studyparticipants/.

56. Wicherts JM, Bakker M, Molenaar D. Willingness to share research data is related to the strength of the evidence and the quality of reporting of statistical results (reproducibility of reported p-values in psychology). PLoS ONE. 2011;6(11):e26828. 